
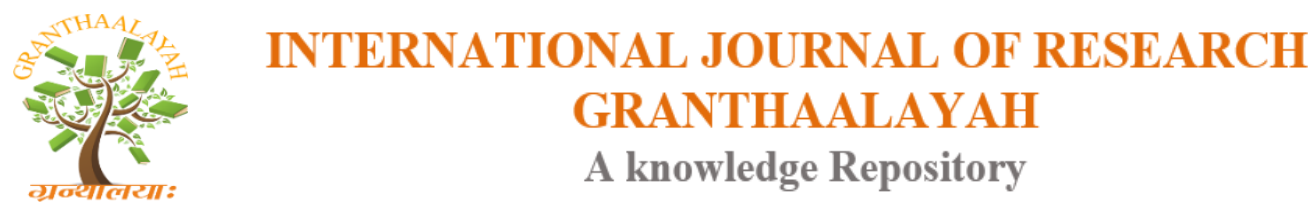

Science

\title{
VIRCATOR - ANALYTICAL AND NUMERICAL ANALYSIS AND OPTIMIZATION OF A VACUUM MICROWAVE HIGH POWER DEVICE
}

\author{
Artur Wymysłowski *1 \\ ${ }^{* 1}$ Wroclaw University of Science and Technology, Faculty of Microsystem Electronics and \\ Photonics, ul. Janiszewskiego 11/17, 50-372 Wroclaw, Poland
}

\begin{abstract}
Vircator is a vacuum microwave device characterised by a pulse working signal and high power. It is classified as a High-Power Microwave (HPM) device and often is a component of Electromagnetic Pulse (EMP) weapons and microwave power transmissions. The direct source of a microwave signal is an oscillation of the so-called virtual cathode. The goal of the presented research was to apply analytical analysis and numerical prototyping methods as a methodology for optimization of the electrical and mechanical design taking into account transformation of electrons' energy into a microwave signal. One of the key drawbacks of viractors as a microwave source is a low energy efficiency transformation, which is typically of a single percentage.
\end{abstract}

Keywords: Vircator; Microwaves; High Power; Numerical Prototyping.

Cite This Article: Artur Wymysłowski. (2018). "VIRCATOR - ANALYTICAL AND NUMERICAL ANALYSIS AND OPTIMIZATION OF A VACUUM MICROWAVE HIGH POWER DEVICE." International Journal of Research - Granthaalayah, 6(5), 47-53. https://doi.org/10.29121/granthaalayah.v6.i5.2018.1422.

\section{Introduction}

Vircator (VIRtual CAthode OscillatOR) is an electronic vacuum microwave device, which is used as a pulse high power microwave source. It is characterized by a simple mechanical structure, lack of a magnetic field and tuneable frequency (typically between 1-10GHz). A comparison of different pulse microwave vacuum devices is given on the figure 1 [1].

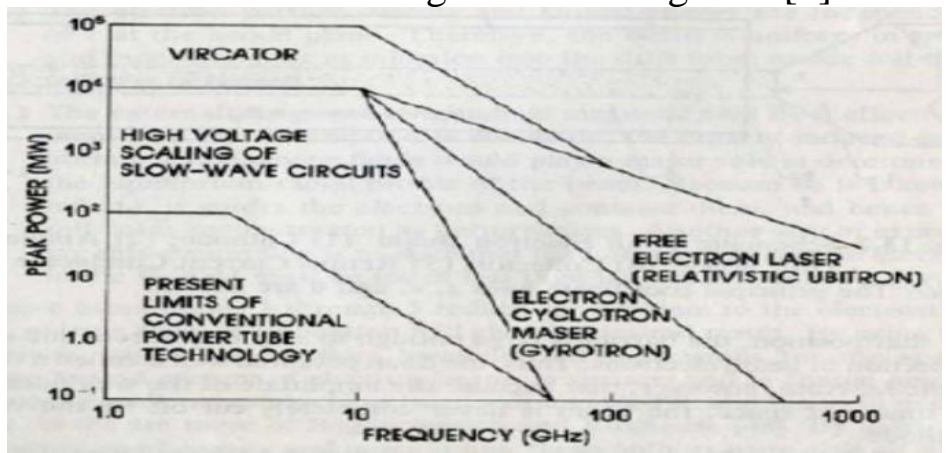

Figure 1: Comparison of different pulse high power microwave vacuum devices [1] 


\section{State-of-the-Art}

Virtual cathode for the first time was observed and described by Irving Langmuire, who analyzed behavior of an electron beam between two electrodes [2]. The mechanical structure of a typical vircator is very simple and can be configured as a triode, which consists of: high power pulse power supply, cathode in a form of an electron emission gun, anode in the form of a transparent grid, cavity region of electrons' oscillations, microwave output system in a form of a waveguide tube. Electrons are emitted from the cathode, then accelerated and transported through the transparent anode. There are three basic designs of a vircator's geometry: axial, reflex, coaxial, which is given schematically in the figure 2 [3].

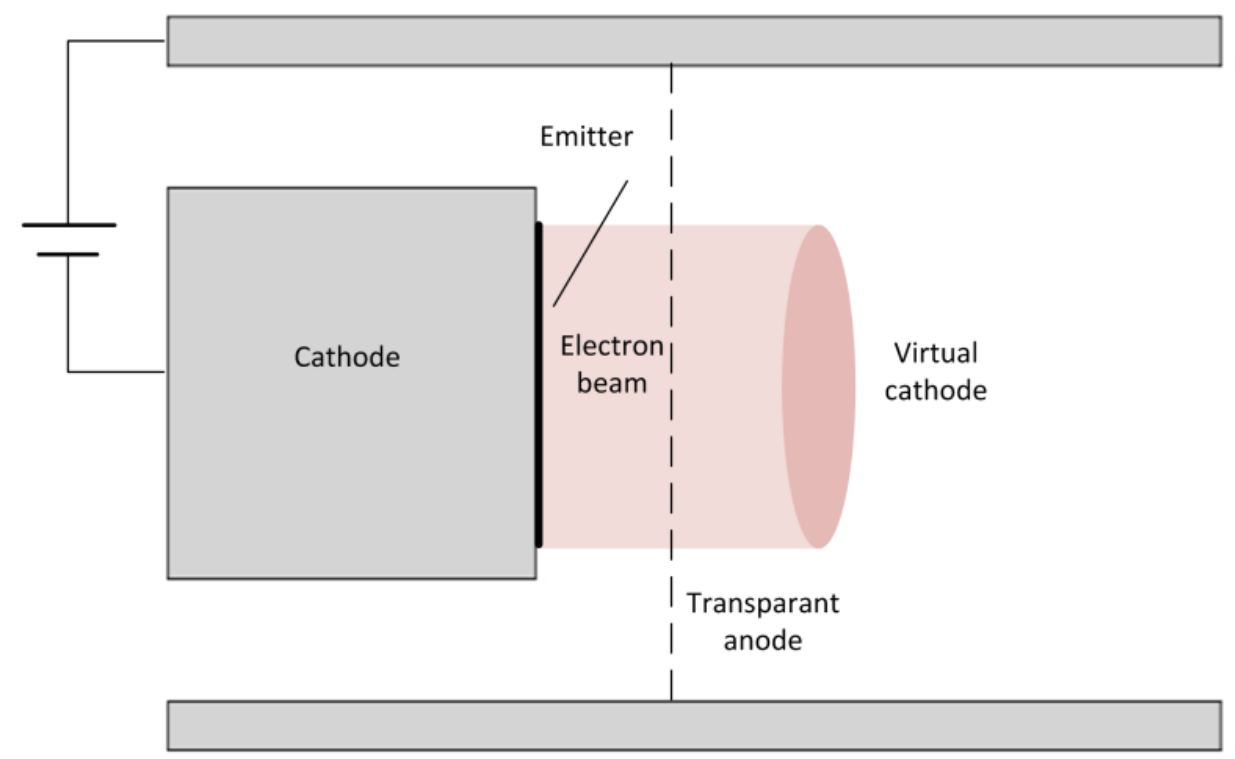

b.
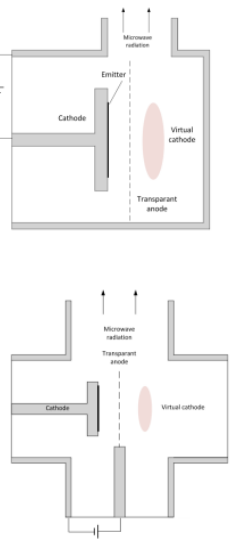

C.

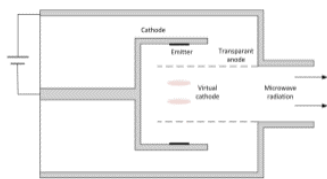

d.

a.

Figure 2: A typical electrical and mechanical structure of a vircator [a] and three basic geometry designs: axial [b], reflex [c], coaxial [d] [3]

The source of a microwave signal are oscillations of a virtual cathode, which is created by a relativistic electron beam being injected into the system of electrodes. It is worth noticing that the oscillations are not due to the relativistic behavior of an electron beam but rather due to the virtual cathode oscillations in time and space. The microwave signal waveform is formed by two phenomena: plasma oscillations and electron flight time between a cathode and a virtual cathode, refereed as the reflexing frequency. Thus the microwave signal is a mixture of the both and it is dominant frequency value is between the plasma frequency $f_{p}$ and $2.5 f_{p}$, as given in the figure 3 [4]. The basic drawback of a vircator is the low efficiency of an energy transformation, which is of the order of a single percentage. This problem seems to be the basic challenge during prototyping. One of the consequences is variety of different configurations and designs [5,6,7]. 


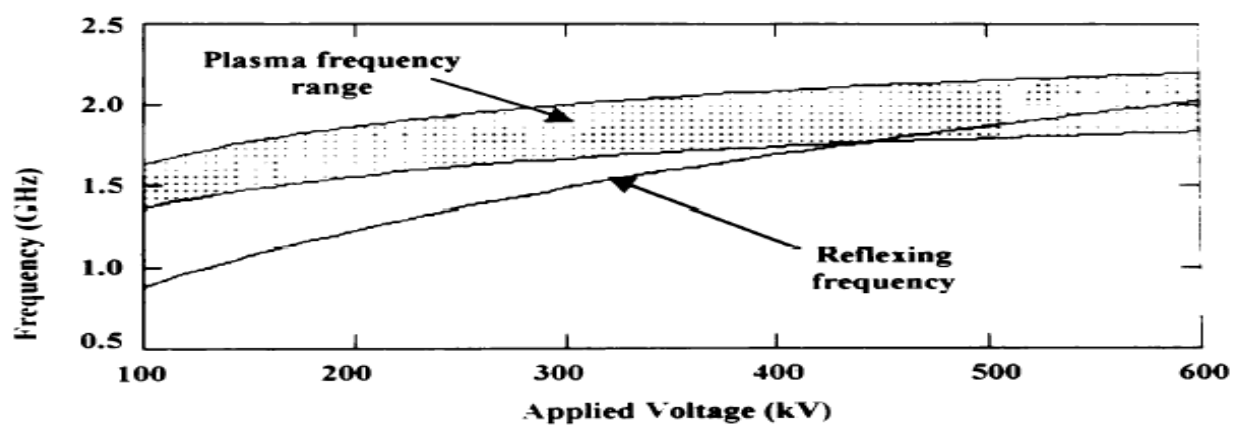

Figure 3: Comparison of a plasma and reflexing frequency in an electron beam with a virtual cathode [4]

\section{Analytical Description and Analysis}

A virtual cathode is formed when the space charge density $\rho$ limit is reached, which leads to a local electric potential minimum, which value is lower than the anode potential $V_{a}$. Therefore, in that region electrons have too low energy to be transported farther and are reversed to the cathode thus causing the electron beam oscillations. The analytical description of such a situation requires solving Poisson's equation:

$$
\nabla^{2} V(x, y, z)=-\frac{\rho_{0}}{\varepsilon_{0}}
$$

Where $V$ is an electrical potential, $\rho_{0}$ is a space charge density of electrons and $\varepsilon_{0}$ is a dielectric constant. A schematic of a virtual cathode creation and an example solution of Poisson's equation assuming non zero space charge density $\rho$ is given in the figure 4 .

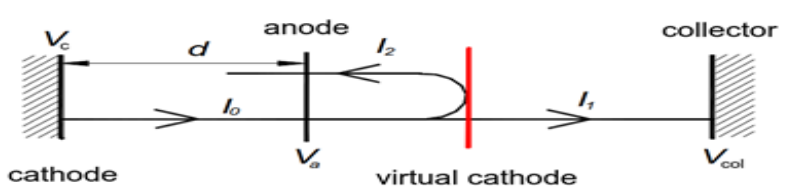

a.

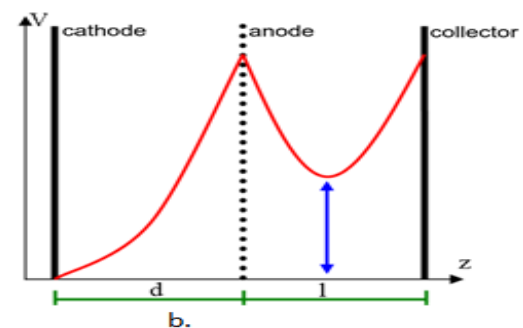

Figure 4: Schematic of a virtual cathode creation (a) and an example solution of Poisson's equation (b)

Using an analytical description a corresponding literature research was performed in order to select the analytical and experimental formulas, which describe the basic vircator's dependence of the input and output parameters, such as: supply voltage $V_{A}$, cathode radius $r_{K}$, anode-cathode distance $d_{A K}$, maximal cathode current $I_{K}$, plasma frequency $f_{p}$, reflexing frequency $f_{r}$, etc. $[2,6,8,9]$. One of the problems concerning the collected formulas was the lack of an appropriate formula for assessing the vircator's energy transformation efficiency and the corresponding output microwave power $P_{m}$. Therefore, a special algorithm based on a quantum-relativistic approach that allowed rough estimation of the above parameters. Additionally, the correction of a plasma $f_{p}$ and reflexing frequency $f_{r}$ due to the relativistic phenomena was included. 
Basing on the analytical formulas and designed algorithm a corresponding analytical vircator calculator was designed using Python software language. The calculator allowed. among others, performing an analytical multi-criteria optimization in order to define an optimal output parameters, e.g.: energy efficiency $\eta$, microwave power $P$, plasma frequency $f_{p}$, reflexing frequency $f_{r}$ as a function of the input parameters: supply voltage $V_{A}$, distance between anode and cathode $d_{A K}$. One of the characteristics of a multicriteria optimization is the lack of one specific optimal solution but existence of a whole set of optimal solution, awhich are equivalent from a mathematical point of view. Most often the optimal solution is selected by assuming some weight coefficients for each output criteria. The example results are given in the figure 5.
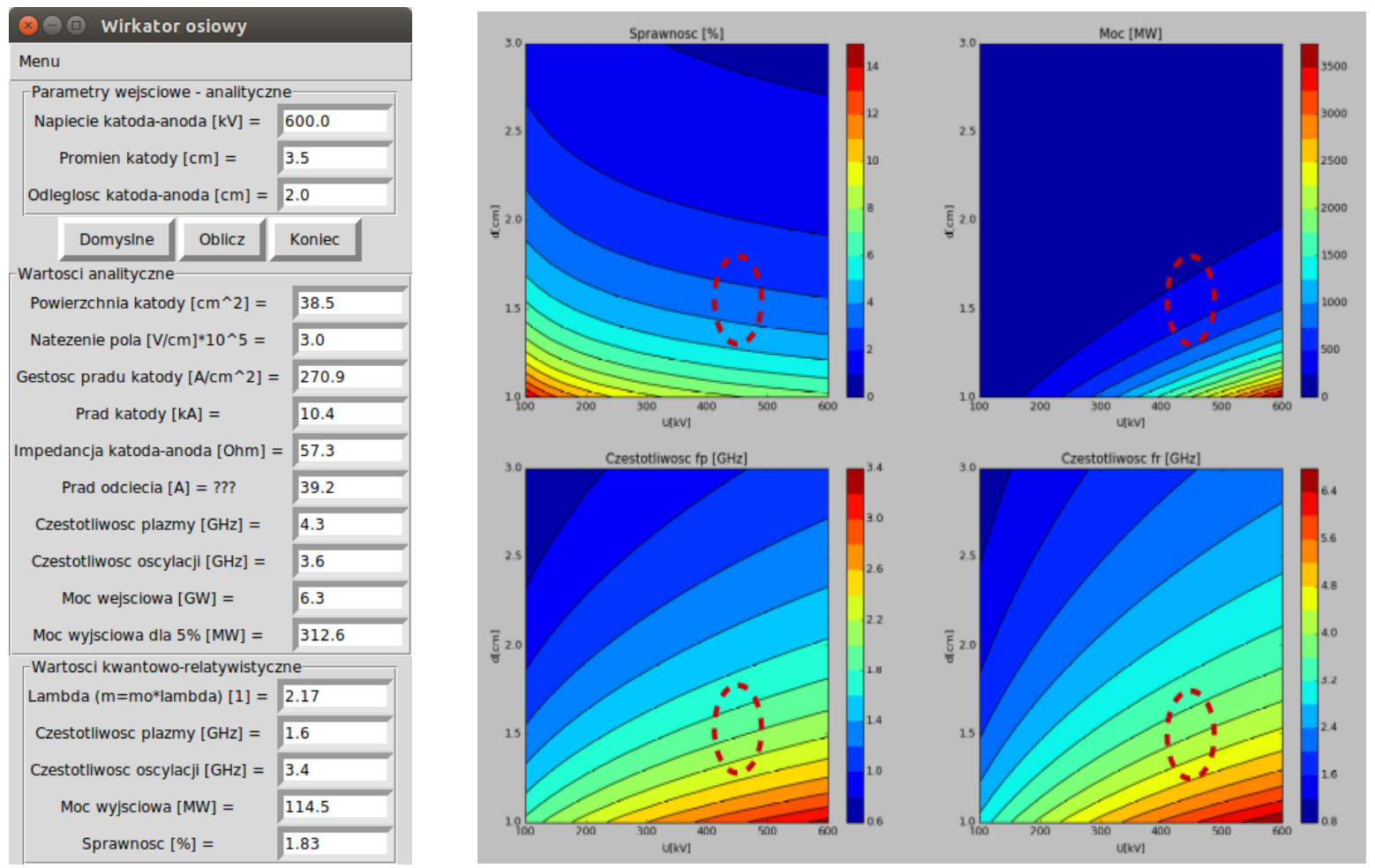

Figure 5: Designed vircator calculator (a) and performed multi-criteria optimization (b) - the optimal region is marked by a dotted red lie (figures are given in the native polish language)

Basing on the achieved results of a multicriteria optimization the initial electrical and geometrical parameters were selected, which in the next step were used for numerical modeling and simulations. The selected key initial input parameters were as follows:

- supply voltage: $U_{A K}=450 \mathrm{kV}$

- distance between anode and cathode: $d_{A K}=15 \mathrm{~mm}$

- cathode radius: $r_{c}=50 \mathrm{~mm}$

According to the designed vircator calculator the expected analytical output parameters should be as follows, as given in the figure 6 :plasma frequency: $f_{p}=2 \mathrm{GHz}$

- reflexing frequency: $f_{r}=4 G H z$

- output microwave power: $P=396 M W$

- energy efficency: $\eta=3.6 \%$ 


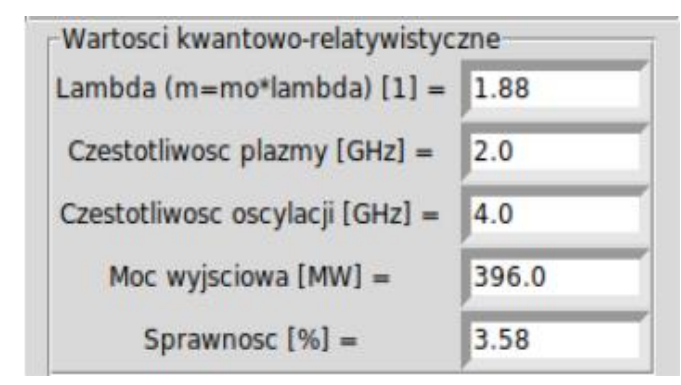

Figure 6: The evaluated analytical results

\section{Numerical Model and Analysis}

Numerical model of the initial vircator was done in CST software package designed for modeling of electromagnetic fields and charged particle trajectories using PIC method (Particle in Cell). The designed numerical model and achieved results are given in the figure 7.

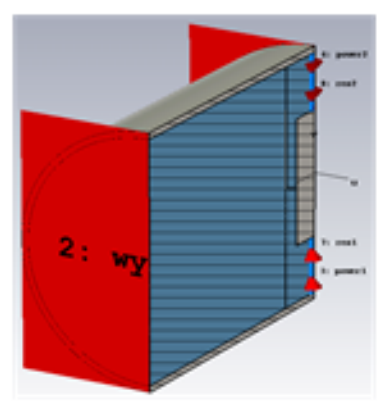

a.

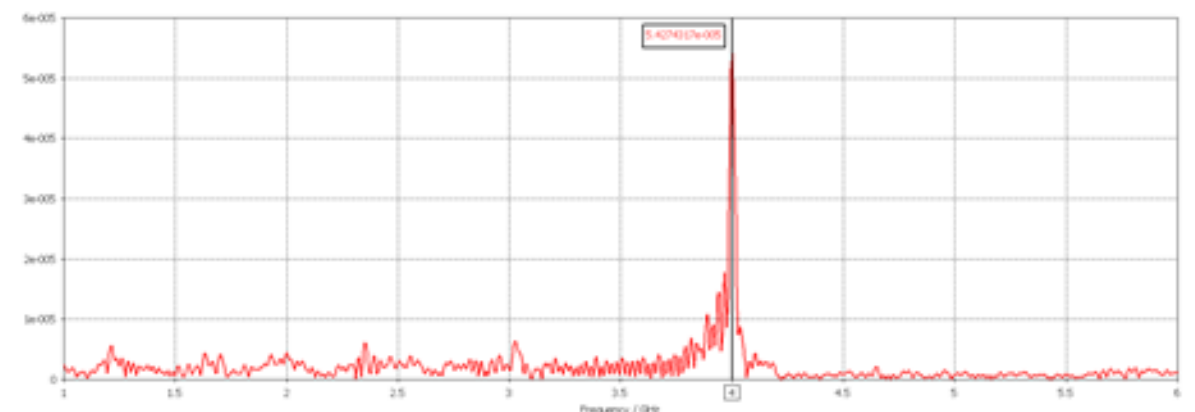

b.

Figure 7: Numerical results of the microwave output frequency spectrum (b) for the designed initial vircator (a)

Numerical modeling results of electron trajectories are presented in the figure $8 \mathrm{~b}$ in a form of a normed momentum $u$, given by a formula

$$
u=\beta \cdot v=\frac{v}{c} \frac{1}{\sqrt{-\left(\frac{v}{c}\right)^{2}}}
$$

where $\beta$ is an electron velocity $v$ in terms of speed of light $c$ and $\gamma$ is Lorentz factor. It should be noted that numerical modeling allows only for assessment of reflexing frequency $f_{r}$ of the microwave signal while plasma frequency $f_{p}$ can only be assessed analytically. Comparing the analytical and numerical results, concerning the reflexing frequency $f_{r}$, it can be concluded that the both analytical and numerical results agree precisely while the energy efficiency is only about $3.6 \%$. Additionally, for the initial vircator, some quantitative and qualitative characteristics were evaluated, which presents the placement of the virtual cathode (Figure 8), and the output microwave signal characteristics (Figure 9). 


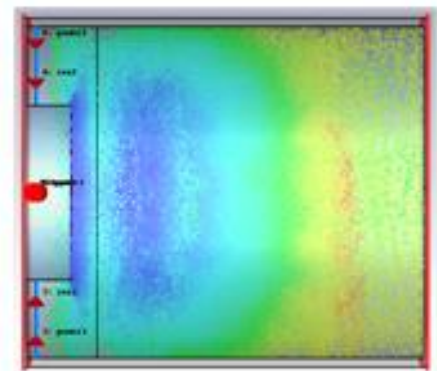

a.

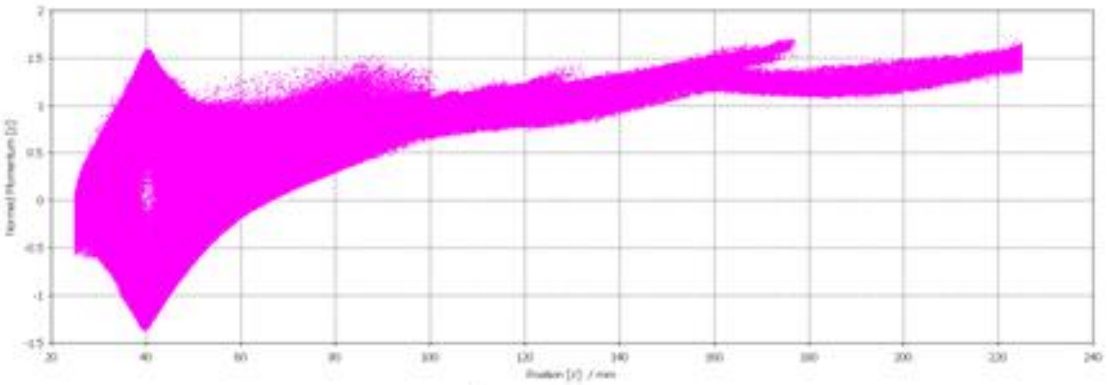

b.

Figure 8: Distribution of an electron energy (a) and scatter of a normed electron momentum (b)
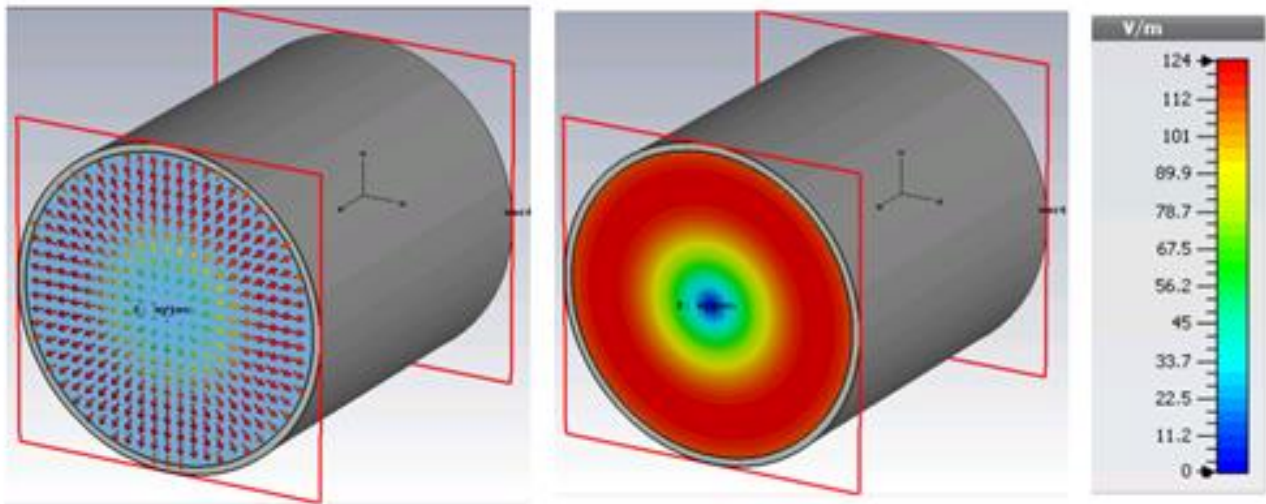

a.
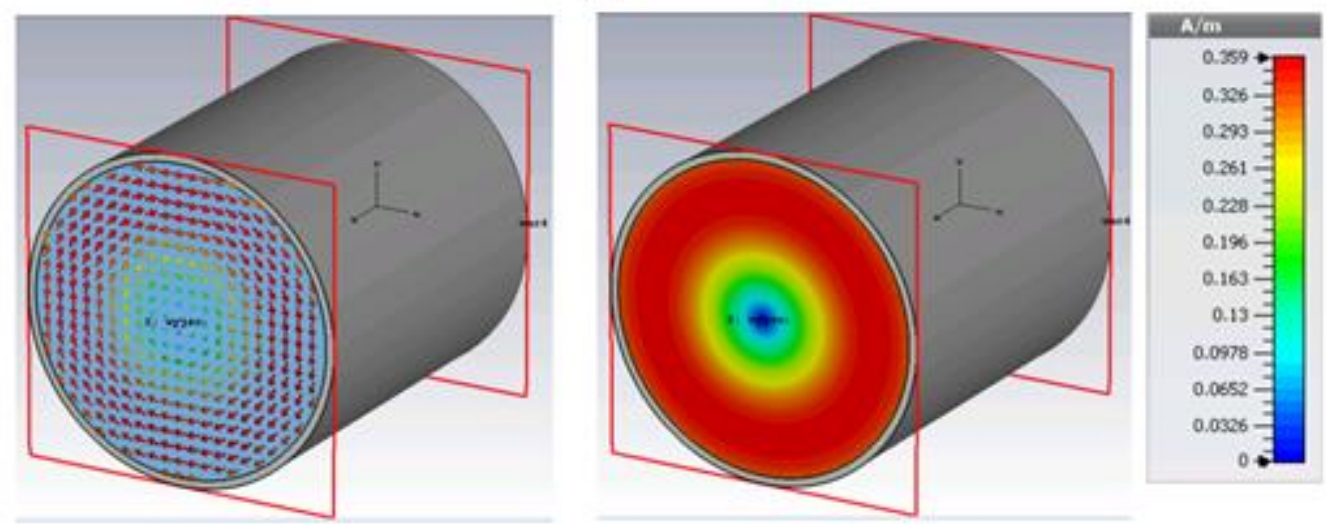

b.

Figure 9: Distribution of a microwave electric (a) and magnetic (b) field (mode 3 TM)

As can be concluded from the presented results of the analytical and numerical analysis the applied methodology can be used for the optimization of a vircator. It should be underlined that the current paper presents only a brief a summary of the first stage of the vircator's electrical and mechanical structure analysis, which finally will aim towards improvement of the transformation of electrons' energy into a microwave signal. One of the key problems, which was noticed is a discrepancy between plasma $f_{p}$ and reflexing frequency $f_{r}$. Unfortunately, during the presented stage of a numerical prototyping, a prototype physical design of a vircator was not yet available, which didn't allowed for an experimental validation of the presented analysis results. 


\section{Conclusions}

This work describes a research, which was devoted to analytical analysis and numerical prototyping methods of a vacuum microwave device refereed as a vircator. One of the key recognized drawbacks of a viractor as a microwave source is a low energy efficiency transformation, which is typically of a single percentage. Therefore, the taken challenge was to recognize the reasons of such a situation and improve the classical viractor's design by using a combined analytical and numerical approach. The achieved results proved that the proposed prototyping methodology seems to be appropriate and the elaborated tools in a form of the analytical viractor's calculator and the corresponding numerical model can be used in the following optimization step. The next step of the presented research would be an application of the results and verified prototyping methodology in order to aligne the plasma $f_{p}$ and reflexing frequency $f_{r}$ by e.g. implementing two anodes instead of a one, etc. It is believed that this will result in an improved energy efficiency of a vircator.

\section{Acknowledgements}

The work was supported by the Statutory Grant of Wroclaw University of Science and Technology (Poland) number 0401/0130/17 in the part concerning application of numerical methods for modeling and simulation of electromagnetic fields and trajectories of charged particles and The Polish National Centre for Research and Development project with number DOB-1-1/1/PS/2014 in the part concerning vircator's mechanical and electrical design optimization.

\section{References}

[1] Yeong-Jer Chen, "Compact, repetitive Marx generator and HPM generation with the vircator", MS Thesis, Texas Tech. Univ. 2005

[2] I. Lanmuir, "The effect of space charge and initial velocities on the potential distribution and thermionic current between parallel plane electrodes", Phys. Rev. 21.419, 1923

[3] C. Möller, "Design and Experiments with High Power Microwave Sources. The Virtual Cathode Oscillator", PhD Thesis, KTH Electrical Engineering, Stockholm, Sweden, 2012

[4] K.S.Woolverton, "High-Power Coaxial Vircator Geometries", Ph.D.Thesis, Texas Tech. Univ. 1998

[5] G. Bedford, "Theory of filamentation in relativistic electron beams", Plasma Phys. 15, 6, 483, 1973

[6] V.L. Granatstein, I.A., Alexeff Eds., "High Power Microwave Sources" - Chapter 13. D.J. Sullivan, J.E. Walsh, E.A. Coutsias, "Virtual Cathode Oscillator (Vircator) Theory", Artech House, Boston, London, 1987

[7] Ki Baek Song, et al., "Output of the axially extracted Virtual cathode oscillator with a cathode wing", IEEE Trans. Plasma Sci.37, 2, 304-310, 2009

[8] C. K. Birdsall, W. B. Bridges, "Electron Dynamics of Diode Regions", Academic Press, New York 1966

[9] W. Czarczynski, "Space-charge Effects in Electron Beams", University of Southampton, C.V.D. Annual Research Report RU13/2/6a, September 1965

*Corresponding author.

E-mail address: artur.wymyslowski@ pwr.edu.pl 